\title{
Non-Diagnostic Q Waves by ECG Finding
}

National Cancer Institute

\section{Source}

National Cancer Institute. Non-Diagnostic Q Waves by ECG Finding. NCI Thesaurus. Code C116134.

An electrocardiographic finding of Q waves that are insufficient for the diagnosis of myocardial infarction. In such cases, a myocardial infarction may be suspected even though ECG diagnostic criteria are absent. (CDISC) 